\section{Innovation methods}

\section{RISK OF SUICIDE BY EMPLOYMENT AND OCCUPATIONAL GROUP: A STUDY USING LINKED DATA FROM THE NATIONAL HEALTH INTERVIEW SURVEY AND THE NATIONAL DEATH INDEX, 2005 - 2015}

${ }^{1}$ Merianne Spencer, ${ }^{1}$ Jonathan Aram, ${ }^{2}$ Matthew Garnett, ${ }^{1}$ Holly Hedegaard. ${ }^{1}$ Centers for Disease Control and Prevention, National Center for Health Statistics; ${ }^{2}$ University of Maryland, College Park

\subsection{6/injuryprev-2020-savir.79}

Statement of Purpose This study evaluates the risk of suicide by employment and occupational group among adults aged 18-64 in the United States using linked data from the National Center for Health Statistics.

Methods Data from the National Health Interview Survey (NHIS) linked to the National Death Index (NDI) was used to examine the risk of suicide among adults ages $18-64$ by employment status and occupational group. Only working-age NHIS respondents with complete occupation information were included. Occupations were categorized by the Census Bureau's Standard Occupational Classification System and grouped as: managerial, professional, teaching/social service, services, sales, and production. Suicides were defined using the International Classification of Diseases, Tenth Revision underlying cause-of-death codes U03, X60-X84, and Y87.0. Cox proportional hazards regression models were fitted to estimate hazard ratios. All estimates incorporate the complex design of the survey.

Results Of the 257,861 sample-adult NHIS participants in 2005 - 2015, 93\% $(239,382)$ did not die, and 7\% $(18,479)$ died by December 31, 2015. Of those who died, 1.3\% (237) were to suicide. Working-age participants in the sales occupational group were at increased risk of suicide $(\mathrm{HR}=1.97,95 \%$ $\mathrm{CI}=1.02-3.80)$ compared to those in the professional occupational group, after controlling for age and sex. Nonemployed participants were at increased risk of suicide compared to employed participants $(\mathrm{HR}=1.57,95 \% \mathrm{CI}=1.06-$ 2.33) after controlling for age and sex.

Conclusions Occupational and employment status may affect risk of suicide. NHIS linked to NDI offers a unique opportunity to examine risk of suicide by both.

Significance and Contributions to Injury and Violence Prevention Science Suicide is the tenth leading cause of death in the U.S., and national studies of suicide by occupation are limited. Understanding suicide by occupation can help inform targeted strategies to prevent suicides in the United States.

\section{Community and youth violence}

\section{INNOVATIVE SOLUTIONS FOR TACKLING URBAN GUN VIOLENCE: PERSPECTIVES FROM THE COMMUNITY ON FIREARM DISPOSAL UNITS}

${ }^{1}$ Douglas Wiebe, ${ }^{1}$ Theresa Soya, ${ }^{2}$ Nicole Thomas, ${ }^{1}$ Omar Koroma, ${ }^{3}$ David Humphreys. ${ }^{1}$ University of Pennsylvania, Penn Injury Science Center; ${ }^{2}$ Thomas Jefferson University; ${ }^{3}$ Department of Social Policy and Intervention, Oxford University

10.1136/injuryprev-2020-savir.80

Statement of Purpose The public stock of firearms in the U.S. is increasing by an estimated three to five million firearms per year. Without enacting unpopular (and unconstitutional) controls on the purchase of firearms, there are few options for limiting the availability of firearms, and reducing rates of physical harm. One approach, which does not infringe on constitutional rights, is voluntary disposal of firearms.

Methods/Approach The Penn Injury Science Center, in collaboration with Oxford University designed and conducted 3 focus groups to: 1) consult members of the community on a local initiatives to provide accessible facilities for disposing of firearms; and 2) understand what design specifications would be essential or desirable for community members. General perceptions around firearm violence and ownership were also assessed. Focus groups were recorded, transcribed and analyzed in NVivo 11, using an iterative process.

Results Sixteen females and fourteen males participated across three focus groups. The majority of participants $(n=23,76 \%)$ were African American with high school $(n=10,33 \%)$, some college $(n=11,37 \%)$ or a college degree $(n=6,17 \%)$ as their highest level of education. Seven primary themes were initially identified and included perceptions towards firearm violence, ownership and laws; distrust of law enforcement; and attitudes, barriers and facilitators for voluntary disposal of firearms. Participants' provided important insights pertaining to the physical appearance, functionality and geographic locations of potential disposal units.

Conclusion Understanding perceptions around the feasibility and acceptability of voluntary disposal of firearms is an important next step towards implementation.

Significance Understanding perceptions around the feasibility and acceptability of voluntary disposal of firearms is an important next step towards implementation. Community input regarding attitudes around firearm ownership and disposal along with considerations on the physical appearance, functionality and geographic locations of firearm disposal units should be integrated into a future pilot demonstration project.

\section{Equity and methods}

\section{DISCORDANCE IN RACE AND ETHNICITY BETWEEN MEDICAL RECORD AND SELF-REPORTED DATA}

${ }^{1}$ Brianna Mills, ${ }^{1}$ Kelsey Conrick, ${ }^{2}$ Danae Dotolo, ${ }^{3}$ Christopher St. Vil, ${ }^{2}$ Megan Moore, ${ }^{1}$ Ali Rowhani-Rahbar. 'University of Washington, Injury Prevention and Research Center; ${ }^{2}$ University of Washington, School of Social Work; ${ }^{3}$ SUNY Buffalo, School of Social Work

\subsection{6/injuryprev-2020-savir.81}

Statement of Purpose Research into race/ethnicity-based injury disparities requires accurate race/ethnicity data. We compared the race/ethnicity documented in electronic medical records (EMRs) against self-report identity to measure the magnitude of EMR misidentification and potential contributing factors.

Approach Descriptive statistics and misclassification matrices were used to assess sensitivity of EMR to true (self-reported) race/ethnicity within two study cohorts (one including all trauma mechanisms $[\mathrm{n}=95]$ and one of firearm assaults $[n=217])$ at a Level-1 trauma center. Both cohorts oversampled people of color. As processes for data collection vary based on how patients arrive, logistic regression models explored associations between arrival type (transfer, EMS, or walk-in) and accurate EMR identification within a subgroup of individuals reporting a single race/ethnicity.

Results Of 276 individuals who self-reported as a single race that mapped to an existing EMR race, only $65.6 \%$ had their 